\title{
The Burkill-Cesari Integral on Spaces of Absolutely Continuous Games
}

\author{
F. Centrone ${ }^{1}$ and A. Martellotti ${ }^{2}$ \\ ${ }^{1}$ Dipartimento di Studi per l'Economia e l'Impresa, Università del Piemonte Orientale, Via Perrone 18, 28100 Novara, Italy \\ ${ }^{2}$ Dipartimento di Matematica e Informatica, Università di Perugia, Via Vanvitelli 1, 06123 Perugia, Italy
}

Correspondence should be addressed to F. Centrone; francesca.centrone@eco.unipmn.it

Received 31 July 2013; Accepted 18 January 2014; Published 10 March 2014

Academic Editor: Jewgeni Dshalalow

Copyright (C) 2014 F. Centrone and A. Martellotti. This is an open access article distributed under the Creative Commons Attribution License, which permits unrestricted use, distribution, and reproduction in any medium, provided the original work is properly cited.

\begin{abstract}
We prove that the Burkill-Cesari integral is a value on a subspace of $A C$ and then discuss its continuity with respect to both the $B V$ and the Lipschitz norm. We provide an example of value on a subspace of $A C$ strictly containing $p N A$ as well as an existence result of a Lipschitz continuous value, different from Aumann and Shapley's one, on a subspace of $A C_{\infty}$.
\end{abstract}

\section{Introduction}

Since the seminal Aumann and Shapley's book [1], it is widely recognized that the theory of value of nonatomic games is strictly linked with different concepts of derivatives. A few papers, up to the recent literature, have investigated these relations (see, e.g., [2-4]). In [1] Aumann and Shapley proved the existence and uniqueness of a value on the space $p N A$, namely, the space spanned by the powers of nonatomic measures (which, under suitable hypotheses, contains, for instance, games of interest in mathematical economics such as transferable utility economies with finite types). Moreover, in $[1$, Theorem $\mathrm{H}]$, the authors provided an explicit formula for the value of games $v$ in $p N A$ in terms of a derivative of their "ideal" set function $v^{*}$.

To the best of our knowledge, the more general contribution so far on the link between derivatives of set functions and value theory is Mertens [3]; his results led to the proof of the existence of a value on spaces larger than $p N A$. A more recent contribution on the same subject is due to Montrucchio and Semeraro [4]. The problem of the existence of a value on the whole space $A C$ of absolutely continuous games (which contains $p N A$ ) is instead still unsolved and challenging. Therefore, proofs of the existence of a value on other subspaces of $A C$, beyond $p N A$, can represent a step forward, and investigations of this kind appear to be in order.
In Epstein and Marinacci [2] the question of the relation between their refinement derivative and the value was posed and a possible direction sketched; in Montrucchio and Semeraro [4], the authors applied their more general (i.e., without the nonatomicity restriction) notion of refinement derivative to the study of the value on certain spaces of games by extending the potential approach of Hart and Mas-Colell [5] to infinite games.

In a previous paper [6] we had pointed out that, in a nonatomic context, the refinement derivative is connected with the classical Burkill-Cesari (BC) integral of set functions and, for BC integrable functions, the BC integral coincides with the refinement derivative at the empty set. Though less general, the $\mathrm{BC}$ integral is analytically more treatable.

Motivated by all these facts, in [6] we have started the study of the BC integral in the framework of transferable utility (TU) games.

In this paper we extend our investigation to develop the connection with the theory of value or of semivalue, also in the light of the problem exposed above. In Section 2 we introduce the general class of BC integrable games and prove that, under natural assumptions, "regular" measure games belong to this class. Moreover, the class of BC integrable games contains a dense subspace of the largely used space $p N A$, where continuous values and semivalues are largely described in the literature (see, for instance, [7]). In addition, 
on the subspace of BC integrable games in $A C$, the BC integral turns out to be a semivalue. Then, as natural, one considers the subspace of feasible BC integrable games, that is, the space where the $\mathrm{BC}$ integral becomes indeed a value. We provide examples of feasible $\mathrm{BC}$ integrable games that do not belong to $p N A$. Actually by means of these examples, we provide a large class of subspaces of $A C$ where the $\mathrm{BC}$ integral is a value, and we also exhibit an example of a subspace of $A C$ strictly containing $p N A$ on which a value can be defined as a sort of direct sum of the usual Aumann-Shapley value and this new set function.

Unfortunately, the $\mathrm{BC}$ integral proves to be not continuous with respect to the $B V$ norm on the $\mathrm{BC}$ integrable games in $A C$. As continuity appears to be a crucial property for many questions concerning the value on subspaces of $B V$, in Section 3 we specialize to the subspace $A C_{\infty} \subset A C$ of the so-called Lipschitz games, where a suitable finer norm (the $\|\cdot\|_{\infty}$-norm) is defined and used as an alternative (see $[8,9]$ ). We completely characterize the scalar measure games (where the measure is nonnegative) that belong to $A C_{\infty}$ and we show that the $\mathrm{BC}$ integral on an appropriate subspace is a Milnor (therefore $\|\cdot\|_{\infty}$-continuous) semivalue. Then again we turn our attention to the subspace of feasible $\mathrm{BC}$ integrable games in $A C_{\infty}$ and to its closure in the $\|\cdot\|_{\infty}$-norm, FEAS ${ }_{\infty}$. In the final part of the paper we consider and somehow characterize the space $F E A S_{\infty} \cap p N A_{\infty}$ (namely, the $\|\cdot\|_{\infty}$-closure of vector measure games generated by polynomials), and we show that the BC integral is not the unique value on it, in that it does not coincide with the Aumann-Shapley value.

\section{A Semivalue on a Space of Burkill-Cesari Integrable Games}

From now on we will denote by $(\Omega, \Sigma)$ a standard Borel space (i.e., $\Omega$ is a Borel set of a Polish space and $\Sigma$ the family of its Borel subsets). $\Omega$ represents a set of players and $\Sigma$ the $\sigma$ algebra of admissible coalitions.

A set function $v: \Sigma \rightarrow \mathbb{R}$ such that $\nu(\varnothing)=0$ is called a transferable utility (TU) game.

For the sake of brevity we refer the reader to $[1,14]$ for the terminology concerning TU games: in particular $B V$ will denote the space of all bounded variation games, endowed with the variation norm $\|\cdot\|_{B V}$. The subspace of nonatomic countably additive measures will be denoted by $N A$ and the cone of the nonnegative elements of $N A$ by $N A^{+}$.

Throughout the paper we will write $\nu \ll \mu$ to mean that absolute continuity by chains holds.

Definition 1 (see [1]). A chain $C$ is a nondecreasing family of sets:

$$
\varnothing=S_{0} \subset S_{1} \subset \cdots \subset S_{n}=\Omega .
$$

A link of a chain is a set of two consecutive elements $\left\{S_{i-1}, S_{i}\right\}$. A subchain of a chain is any set of links.
A chain will be identified with the subchain consisting of all the links. Given a game $v$ and a subchain $\Lambda$ of a chain $\mathrm{C}$, the variation of $v$ over $\Lambda$ is defined as

$$
\|v\|_{\Lambda}:=\sum\left|v\left(S_{i}\right)-v\left(S_{i-1}\right)\right|
$$

where the sum ranges over all indexes $i$ such that $\left\{S_{i-1}, S_{i}\right\}$ is a link in the subchain.

Definition 2 (see [1]). If $\nu$ and $w$ are two games defined on $\Sigma$, $v$ is said to be absolutely continuous with respect to $w$ if for every $\varepsilon>0$ there exists a $\delta>0$ such that, for every chain $C$ and every subchain $\Lambda$ of $C$,

$$
\|w\|_{\Lambda} \leq \delta \Longrightarrow\|\nu\|_{\Lambda} \leq \varepsilon .
$$

The space $A C \subset B V$ introduced by Aumann and Shapley [1] is the space of all games $\nu$ for which there exists $\mu \epsilon$ $N A^{+}$such that $\nu$ is absolutely continuous with respect to $\mu$.

We also refer the reader to [2] and to our previous paper [6] for details about the Epstein-Marinacci refinement derivative.

A partition $D$ of a set $E \in \Sigma$ is a finite family of pairwise disjoint elements of $\Sigma$, whose union is $E$. By $\Pi(E)$ we will denote the set of all the partitions of $E$. A partition $\bar{D} \in \Pi(E)$ is a refinement of another partition $D \in \Pi(E)$ if each element of $D$ is union of elements of $\bar{D}$.

As in [11], given a monotone nonatomic game $\lambda$ one defines the mesh of a partition $D$ as

$$
\delta_{\lambda}(D)=\max \{\lambda(I), I \in D\}
$$

and the Burkill-Cesari (BC) integral of a game $v$ with respect to $\delta_{\lambda}$ as

$$
E \longmapsto \int_{E} \nu=\lim _{\substack{\delta_{\lambda}(D) \rightarrow 0 \\ D \in \Pi(E)}} \sum_{I \in D} \nu(I)
$$

We denote by $\mathrm{BC}$ the space of games $v$ such that there exists $\lambda \in N A^{+}$so that $\nu$ is $\mathrm{BC}$ integrable with respect to the mesh $\delta_{\lambda}$.

The BC integral does not depend upon the integration mesh (see Proposition 5.2 in [6]); in other words, for every $\lambda \in N A^{+}$such that $v$ is $\delta_{\lambda}$ - $\mathrm{BC}$ integrable, the $\mathrm{BC}$ integral is the same. Moreover, the $\mathrm{BC}$ integral of a game $v$ is a finitely additive measure and, as observed in [6], it coincides with the Epstein-Marinacci outer derivative at the empty set $\partial_{\varnothing}^{+}(\nu, \cdot)$ (see [2]). Hence, from now on we will use the notation $\partial_{\varnothing}^{+}(\nu, \cdot)$.

As we will see, the space BC contains many games which are of interest in the literature: we begin by recalling a sufficient condition for vector measure games to be in $\mathrm{BC}$, which is an immediate consequence of [6, Theorem 6.1].

Proposition 3. Let $P: \Sigma \rightarrow \mathbb{R}^{n}$ be a nonatomic vector measure, and let $f: \mathbb{R}^{n} \rightarrow \mathbb{R}$ be a function with $f(0)=0$. If $f$ is differentiable at 0 , then the game $\nu=f \circ P \in B C$, and $\partial_{\varnothing}^{+}(\nu, F)=\nabla f(0) \cdot P(F), F \in \Sigma$.

Anyway, the class of BC integrable games is not limited to smooth measure games. Indeed note that the converse implication of the previous proposition does not hold: consider as 
in $[6$, Example 3.2] $f: \mathbb{R} \rightarrow \mathbb{R}$ to be any discontinuous solution to the functional equation

$$
f(x+y)=f(x)+f(y), \quad x, y \in \mathbb{R} .
$$

Then $v=f \circ P$ is additive, and therefore for each $F \in \Sigma$ and each $D \in \Pi(F)$ one has

$$
\sum_{I \in D} \nu(I)=\sum_{I \in D} f[P(I)]=f[P(F)]=\nu(F)
$$

and hence $v$ is BC integrable with respect to $\delta_{P}$ although $f$ is not differentiable at 0 .

As for the relation between the spaces $A C$ and $\mathrm{BC}$, it is well known (see Theorem C of [1]) that the game $v=\sqrt{\lambda}$ (with $\lambda$ the Lebesgue measure on $[0,1]$ ) belongs to $A C$. Anyway $\nu \notin$ BC. To see it, one has to show that $v$ is not BC integrable with respect to any mesh $\delta_{\mu}$ determined by some $\mu \in N A^{+}$. Indeed $\nu$ is not refinement differentiable at $\varnothing$, and then it cannot be $\mathrm{BC}$ integrable with respect to $\delta_{\mu}$; to get convinced that $\nu$ does not admit outer refinement derivative at $\varnothing$, observe that for every partition $D_{o} \in \Pi(\Omega)$ and every $\delta>0$ we can provide a refinement $D^{\prime}=\left\{I_{1}, \ldots, I_{k}, I_{o}\right\}$ such that $\lambda\left(I_{1}\right)=\cdots=\lambda\left(I_{k}\right)$ and $\lambda\left(I_{o}\right)<\delta[15$, Lemma 3.5]. Clearly we can choose $k$ quite larger than say $\sharp D_{o}$. Also we can choose $\delta=\delta(\varepsilon)$ determined by the uniform continuity of $x \mapsto \sqrt{x}$ on $[0,1]$. Thus

$$
\left|\sum_{I \in D^{\prime}} \nu(I)-\sqrt{k(1-\delta)}\right|<\varepsilon
$$

which shows that the refinement limit does not exist.

In fact $\lambda\left(\Omega \backslash I_{o}\right)>1-\delta$, and therefore $\lambda\left(I_{j}\right)>((1-$ $\delta) / k), j=1, \ldots, k$, whence $v\left(I_{j}\right) \geq \sqrt{((1-\delta) / k)}, j=1, \ldots, k$, while $\nu\left(I_{o}\right)<\varepsilon$.

So

$$
\begin{aligned}
k \cdot \frac{\sqrt{1-\delta}}{\sqrt{k}} & \leq \sum_{I \in D^{\prime}} \nu(I)=\nu\left(I_{o}\right)+\sum_{j=1}^{k} \nu\left(I_{j}\right) \\
& \leq \varepsilon+k \cdot \frac{\sqrt{1-\delta}}{\sqrt{k}} .
\end{aligned}
$$

On the contrary, any strongly nonatomic finitely additive measure $v$ that is not countably additive provides an example of game belonging to $\mathrm{BC}$ (for $\partial_{\varnothing}^{+}(\nu, \cdot)=\nu(\cdot)$ ), but not to $A C$ (see the different notions of absolute continuity in [6]).

Therefore, we can now consider the space $V=\mathrm{BC} \cap A C$.

The following result states that the same measure can be used for the absolute continuity and the BC integrability of a game in $V$.

Proposition 4. The space $V$ can be equivalently defined as the space of games $v$ such that there exists $\mu \in N A^{+}$such that $\nu \ll \mu$ and $\nu$ is $B C$ integrable with respect to $\delta_{\mu}$.

Proof. The fact that each game $\nu$ for which there exists $\mu \epsilon$ $N A^{+}$such that $\nu \ll \mu$ and $\nu$ is BC integrable with respect to $\delta_{\mu}$ lies in $V$ is straightforward.

Conversely, let $v \in V$; then there are $\mu_{1}, \mu_{2} \in N A^{+}$such that $v \ll \mu_{1}$ (since $\left.v \in A C\right)$ and $\nu$ is $\delta_{\mu_{2}}$-BC integrable.
Then consider $\mu=\mu_{1}+\mu_{2}$; evidently $\nu \ll \mu$ and, in view of Proposition 5.2 in [6], $\nu$ is also $\delta_{\mu}$-BC integrable.

From [1] we recall the following.

Definition 5. Let $\mathscr{G}$ denote the space of automorphisms of $(\Omega, \Sigma)$, then each $\vartheta \in \mathscr{G}$ induces a linear mapping $\vartheta_{*}$ of $B V$ onto itself, defined by

$$
\left(\vartheta_{*} \nu\right)(E)=\nu(\vartheta(E))
$$

for $E \in \Sigma$. A subspace that is invariant under $\vartheta_{*}$ for every $\vartheta \in \mathscr{G}$ is called symmetric.

Proposition 6. The space $V$ is symmetric.

Proof. We need to prove that for every $\vartheta \in \mathscr{G}$ and every game $\nu \in V$ the game $\vartheta_{*} \nu$ defined in (10) is in $V$; namely, it is « with respect to some nonatomic measure, and it is $\mathrm{BC}$ integrable too.

Let $\mu$ be a measure in $N A^{+}$with respect to which we have $\nu \ll \mu$ and $\nu$ is $\delta_{\mu}$-BC integrable (thanks to Proposition 4 we can always assume that the default measure is the same).

Fix $\vartheta$; note that $\vartheta$ preserves set operations; therefore easily $\lambda=\vartheta_{*} \mu$ is in $N A^{+}$.

It is also immediate to check that $\vartheta_{*} \nu \ll \lambda$, because $\vartheta$ transforms chains and subchains into chains and subchains as well.

It remains to prove that $\vartheta_{*} \nu$ is BC integrable with respect to the mesh $\delta_{\lambda}$. Indeed we will prove that

$$
\partial_{\varnothing}^{+}\left(\vartheta_{*} \nu, F\right)=\partial_{\varnothing}^{+}(\nu, \vartheta(F))
$$

for every $F \in \Sigma$.

To this aim, for any $F \in \Sigma$ and any $\varepsilon>0$ fixed, one has to find $\delta(\varepsilon, F)>0$ such that for every partition $D \in \Pi(F)$ with $\delta_{\lambda}(D)<\delta$ there holds

$$
\left|\sum_{I \in D} \vartheta_{*} \nu(I)-\partial_{\varnothing}^{+}(\nu, \vartheta(F))\right|<\varepsilon .
$$

Since $v$ is BC integrable, to each $\varepsilon>0$ there corresponds $\tau(\varepsilon, \vartheta(F))>0$ such that for each partition $D \in \Pi[\vartheta(F)]$ with $\delta_{\mu}(D)<\tau$ there follows

$$
\left|\sum_{J \in D} \nu(J)-\partial_{\varnothing}^{+}(\nu, \vartheta(F))\right|<\varepsilon .
$$

Clearly we can rewrite (12) as

$$
\left|\sum_{I \in D} \nu[\vartheta(I)]-\partial_{\varnothing}^{+}(\nu, \vartheta(F))\right|<\varepsilon .
$$

We choose $\delta(\varepsilon, F)=\tau(\varepsilon, \vartheta(F))$; thus if $D \in \Pi(F)$ has $\delta_{\lambda}(D)<$ $\delta$, the corresponding partition $D^{\prime}=\{\vartheta(I), I \in D\} \in \Pi[\vartheta(F)]$ 
has $\delta_{\mu}\left(D^{\prime}\right)<\delta=\tau$ since, for each $I \in D$, clearly $\lambda(I)=$ $\vartheta_{*} \mu(I)=\mu[\vartheta(I)]<\delta=\tau$ and hence

$$
\begin{aligned}
& \left|\sum_{I \in D} \nu[\vartheta(I)]-\partial_{\varnothing}^{+}(\nu, \vartheta(F))\right| \\
& \quad=\left|\sum_{J \in D^{\prime}} \nu(J)-\partial_{\varnothing}^{+}(\nu, \vartheta(F))\right|<\varepsilon .
\end{aligned}
$$

According to [7] we recall the following definition.

Definition 7. A linear mapping $\varphi: \mathscr{Y} \rightarrow N A$ on a symmetric subspace $\mathcal{Y}$ of $B V$ is called a semivalue provided that it satisfies the following properties:

V.1 (symmetry): $\vartheta_{*} \varphi=\varphi\left(\vartheta_{*}\right)$ for each $\vartheta \in \mathscr{G}$;

V.2 (positivity): $\varphi$ is positive, that is for every monotone game $v$ the measure $\varphi(\nu)$ is nonnegative;

V.3 (projection axiom): $\varphi$ is the identity operator on $N A \cap$ y;

when $\varphi$ satisfies also the property:

V.4 (efficiency): for each $\nu \in \mathcal{Y}$ there holds $\varphi(\nu)(\Omega)=$ $\nu(\Omega)$;

$\varphi$ is called a value on $\mathcal{Y}$ (compare with [1]).

The following result immediately derives from (11) and the definition of $\partial_{\varnothing}^{+}$.

Corollary 8. The mapping $\partial_{\varnothing}^{+}: V \rightarrow N A$ is a semivalue on $V$.

Consider now the space $F E A S_{0}=\left\{\nu \in \mathrm{BC}: \partial_{\varnothing}^{+}(\nu, I)=\right.$ $\nu(I)\}$ and define the space FEAS $=F E A S_{0} \cap V=F E A S_{0} \cap A C$. Obviously $\partial_{\varnothing}^{+}$is a value on FEAS.

The next example shows that this space contains several games which do not belong to $p N A$, and through these one finds several new subspaces of $A C$ on which $\partial_{\varnothing}^{+}$defines a value.

Example 9. Let $\mu$ be a signed measure on a measurable space $(\Omega, \Sigma)$ with range say $[-1,1]$ and, for instance, $\mu(\Omega)=\alpha \epsilon$ ] $0,1[$, and let $f:[-1,1] \rightarrow \mathbb{R}$ be defined as

$$
f(x)= \begin{cases}-\alpha^{2} & \text { if } x \in[-1,-\alpha[ \\ \alpha x & \text { if } x \in[-\alpha, \alpha] \\ \alpha^{2} & \text { if } x \in] \alpha, 1] .\end{cases}
$$

Let $\nu=f \circ \mu$ and consider the space $E=\operatorname{span}\left\{\vartheta^{*} v: \vartheta \in \mathscr{G}\right\}$. Then $\nu \in A C \backslash p N A$ so that $E \subset A C$ but $E \not \subset p N A$ and $\partial_{\varnothing}^{+}$is a value on $E$ :

Fact I. $v \in F E A S_{0}$; indeed, since $f^{\prime}(0)$ exists, by Proposition 3 , $\nu \in \mathrm{BC}$ and $\partial_{\varnothing}^{+}(\nu, \cdot)=\alpha \mu$ whence immediately $\partial_{\varnothing}^{+}(\nu, \Omega)=$ $\alpha^{2}=v(\Omega)$.

Fact II. $v \notin p N A$; indeed, according to Kohlberg [13] a measure game $f \circ \mu$, where $\mu$ is a signed measure, is in $p N A$ if and only if the function $f$ is continuously differentiable in ]$-1,1[$.

Fact III. $v \in A C$; for the proof, see the Appendix.

Finally $E$ is symmetric, and by linearity and relationship (11), $\partial_{\varnothing}^{+}$is efficient on $E$; therefore it is a value on $E$.

It is clear that one can use functions of different forms to provide classes of measure games $f \circ \mu$ with signed $\mu$ that are in $A C \backslash p N A$ and in $F E A S_{0}$, and hence similar subspaces of $A C$ on which $\partial_{\varnothing}^{+}$is a value. These subspaces will not be contained in $p N A$ because the generating game $\nu \notin p N A$. However, we can go a little further; in fact the next example shows that, by means of a similar construction, there are subspaces in $A C$ strictly containing $p N A$ on which a value can be defined.

Example 10. On $\Omega=[-1,1]$ equipped with the usual Borel $\sigma$-algebra, consider the signed measure $\mu(S)=\int_{S}(\operatorname{sign} x) d x$.

Denote $\left.I_{1}=[-1,-(1 / 2)], I_{2}=\right]-(1 / 2),(1 / 2)\left[\right.$, and $I_{3}=$ $[(1 / 2), 1]$ and take the function $f: \Omega \rightarrow \mathbb{R}$ defined by

$$
f(x)= \begin{cases}-x-\frac{1}{2} & \text { if } x \in I_{1} \\ 0 & \text { if } x \in I_{2} \\ x-\frac{1}{2} & \text { if } x \in I_{3}\end{cases}
$$

Define the scalar measure game $\nu=f \circ \mu$ and take then $w=$ $\nu+\lambda$, where $\lambda$ denotes the usual Lebesgue measure.

As in the previous example, $v$ and hence $w$ are in $F E A S_{0}$, $w \notin p N A$ (for $v \notin p N A$ ) and $w \in A C$ (again, for the proof, see the Appendix).

Furthermore $v$ (and hence $w$ ) is in $p N A^{\prime}$, since $f$ is continuous [16].

Thus $v \in F E A S \backslash p N A$.

Again take $E=\operatorname{span}\left\{\vartheta^{*} w: \vartheta \in \mathscr{G}\right\}$ and denote by $p N A E$ the smallest linear subspace containing $p N A$ and $E$. Define $\Psi: p N A E \rightarrow N A$ as follows: for each $\gamma=\sum_{i=1}^{m} c_{i} \vartheta_{i}^{*} w+p \epsilon$ $p N A E$, set $\Psi(\gamma)=\sum_{i=1}^{m} c_{i} \partial_{\varnothing}^{+}\left(\vartheta_{i}^{*} w, \cdot\right)+\phi_{\text {AS }}(p)$, where $\phi_{\text {AS }}$ is the usual Aumann and Shapley value.

$\Psi$ is a value on $p N A E$ (see the Appendix for the proof).

Finally, the following example shows that $\partial_{\varnothing}^{+}$is not continuous on $V$ equipped with the variation norm.

Example 11. Consider the sequence of scalar measure games $v_{n}=f_{n} \circ P$, where $f_{n}(x)=\min \{x, 1 / n\}$ and $P \in N A^{1}$. Then $\left\|\nu_{n}\right\|_{B V} \rightarrow 0$ as $n \rightarrow+\infty$. However, $\partial_{\varnothing}^{+}\left(\nu_{n}, S\right)=f_{n}^{\prime}(0) P(S)=$ $P(S)$ does not converge to 0 .

\section{The Operator $\partial_{\varnothing}^{+}$on Subspaces of Lipschitz Games}

In [9] the author considers the class $A C_{\infty}$ of Lipschitz games, that is, games $\nu$ in $B V$ for which there exists a measure $\mu \epsilon$ $N A^{+}$such that both $\mu-\nu$ and $\nu+\mu$ are monotone games. The reason why these games are called Lipschitz is the fact that the 
condition can be equivalently labelled in the following form: for every link $S \subset T$ in $\Sigma$ there holds

$$
|\nu(T)-\nu(S)| \leq \mu(T)-\mu(S) .
$$

The connection to the Lipschitz condition is made even stronger by the following.

Proposition 12. For a scalar measure game $v=f \circ \lambda$ the following are equivalent:

(1) $v \in A C_{\infty}$;

(2) $f$ is Lipschitz on the interval $[0, \lambda(\Omega)]$ (with $\mu(\Omega)$ as Lipschitz constant, for each $\mu \in N A^{+}$for which (18) above is satisfied);

(3) (18) holds for $\mu=L \lambda$, with L Lipschitz constant for $f$.

Proof. To prove that (1) implies (2), assume that $\nu=f \circ \lambda$ is a Lipschitz game, and let $\mu \in N A^{+}$be a measure for which (18) holds. For simplicity we assume that $\lambda$ is a probability measure. Let $t, t^{\prime} \in[0,1]$ with say $t<t^{\prime}$.

Then, by Lyapunov theorem, there exist sets $\Omega_{t} \subset \Omega_{t^{\prime}} \subset \Omega$ such that

$$
\begin{gathered}
(\lambda, \mu)\left(\Omega_{t}\right)=t(\lambda, \mu)(\Omega), \\
(\lambda, \mu)\left(\Omega_{t^{\prime}}\right)=t^{\prime}(\lambda, \mu)(\Omega) .
\end{gathered}
$$

Hence $f(t)=f\left[\lambda\left(\Omega_{t}\right)\right]=v\left(\Omega_{t}\right)$ and $f\left(t^{\prime}\right)=\nu\left(\Omega_{t^{\prime}}\right)$; then from (18)

$$
\begin{aligned}
\left|f(t)-f\left(t^{\prime}\right)\right| & =\left|\nu\left(\Omega_{t}\right)-\nu\left(\Omega_{t^{\prime}}\right)\right| \leq \mu\left(\Omega_{t} \backslash \Omega_{t^{\prime}}\right) \\
& =\left(t^{\prime}-t\right) \mu(\Omega)
\end{aligned}
$$

The fact that a Lipschitz function $f$ generates a Lipschitz game (where one can precisely choose $L \lambda=\mu$ in (18)) is immediate, so (2) implies (3).

Also (3) implies (1) trivially.

It is immediate to note that $A C_{\infty} \subset A C$. However, the smaller space can be equipped with an alternative norm defined in the following way; for every $\mu \in N A^{+}$such that (18) holds, write $-\mu \preceq \nu \preceq \mu$. Then we set

$$
\|\nu\|_{\infty}=\inf \left\{\mu(\Omega), \mu \in N A^{+},-\mu \preceq \nu \preceq \mu\right\} .
$$

Then $A C_{\infty}$ is a Banach space when equipped with the above norm.

Again from [9] we quote the following definition.

Definition 13. Let $v \in A C_{\infty}$ and define the following two subsets of $N A$ :

$$
\begin{aligned}
& D^{\nu}=\{\lambda \in N A \mid \nu \preceq \lambda\}, \\
& D_{\nu}=\{\lambda \in N A \mid \lambda \preceq \nu\} .
\end{aligned}
$$

Then the following two measures exist: $v^{*}=$ g.l.b. $D^{\nu}, v_{*}=$ l.u.b. $D_{v}$, and immediately $v_{*} \leq v^{*}$ (although the symbol $\leq$ should be distinguished from $\preceq$, as the first one refers to setwise ordering while the second one to the order induced by the cone of monotonic games, in the case of measures they actually assume the same meaning).

Let $N A \subseteq Q \subseteq A C_{\infty}$ be a linear subspace, and let $\psi$ : $Q \rightarrow N A$ be a linear operator; we will say that $\psi$ is a Milnor operator $(\mathrm{MO})$ provided that for every $\nu \in Q$ we have

$$
v_{*} \leq \psi v \leq v^{*} .
$$

Consider now the vector subspace $Q=B C \cap A C_{\infty}$ of Lipschitz games that are $\mathrm{BC}$ integrable.

$\mathrm{Q}$ is strictly included in $\mathrm{BC}$, for there are easy examples of games in $\mathrm{BC} \backslash A C_{\infty}$.

For instance, consider the function $f:[0,1] \rightarrow \mathbb{R}$ defined as

$$
f(x)= \begin{cases}x & \text { if } 0 \leq x \leq \frac{\sqrt{2}}{2} \\ \sqrt{1-x^{2}} & \text { if } \frac{\sqrt{2}}{2} \leq x \leq 1\end{cases}
$$

and the scalar measure game $\nu=f \circ \lambda$, where $\lambda$ represents the usual Lebesgue measure. Then $v \in \mathrm{BC}$ with $\partial_{\varnothing}^{+}(\nu)=$ $f^{\prime}(0) \lambda=\lambda$ thanks to Proposition 3, but $\nu \notin A C_{\infty}$ since $f$ is not Lipschitz on $[0,1]$.

Also the inclusion $Q \subset A C_{\infty}$ is a strict one, for there are Lipschitz games that are not in BC. To see this we need the following result, which is a partial converse of Proposition 3.

Proposition 14. Let the scalar measure game $\nu=f \circ \mu, \mu \in$ $N A^{+}, \mu \neq 0$ be in $A C_{\infty}$; then the following are equivalent:

(1) $f$ admits right-hand side derivative at 0 ;

(2) $\nu$ is $\delta_{\mu}$-BC integrable and hence $v \in Q$.

Proof. The implication (1) $\Rightarrow$ (2) follows from Proposition 3.

We turn then to the implication $(2) \Rightarrow(1)$.

As $v \in Q$, there exists $\lambda \in N A^{+}$such that $v$ is $\delta_{\lambda^{-}}$ BC integrable. Since $v \in A C_{\infty}$ we already know that $f$ is Lipschitz; hence the ratios $f(x) / x$ are bounded. Assume by contradiction that $f^{\prime}(0)$ does not exist. Then it can only happen that

$$
-\infty<\ell_{1}=\liminf _{x \rightarrow 0} \frac{f(x)}{x}<\limsup _{x \rightarrow 0} \frac{f(x)}{x}=\ell_{2}<+\infty .
$$

Choose then two decreasing sequences $\left.\left.\left\{x_{n}^{\prime}\right\},\left\{x_{n}^{\prime \prime}\right\} \in\right] 0, \mu(\Omega)\right]$ with $\lim _{n} x_{n}^{\prime}=\lim _{n} x_{n}^{\prime \prime}=0$ and

$$
\lim _{n} \frac{f\left(x_{n}^{\prime}\right)}{x_{n}^{\prime}}=\ell_{1}, \quad \lim _{n} \frac{f\left(x_{n}^{\prime \prime}\right)}{x_{n}^{\prime \prime}}=\ell_{2} .
$$

Fix $F \in \Sigma$ with $\mu(F)>0$ and $\varepsilon \in] 0, \mu(F)]$. Then there exists $\bar{n} \in \mathbb{N}$ such that for each $n>\bar{n}$

$$
\begin{aligned}
& \left|\frac{f\left(x_{n}^{\prime}\right)}{x_{n}^{\prime}}-\ell_{1}\right|<\frac{\varepsilon}{3 \mu(F)}, \\
& \left|\frac{f\left(x_{n}^{\prime \prime}\right)}{x_{n}^{\prime \prime}}-\ell_{2}\right|<\frac{\varepsilon}{3 \mu(F)} .
\end{aligned}
$$


By means of the continuity of $f$ at 0 , choose next $\tilde{n}>\bar{n}$ such that $|f(x)|<\varepsilon / 3$ whenever $x \leq x_{\tilde{n}}^{\prime}$; also $\widetilde{n}$ can be chosen so that $\left|\ell_{1}\right| x_{\widetilde{n}}^{\prime}<\varepsilon / 3$ and such that $x_{\widetilde{n}}^{\prime}(\lambda(F) / \mu(F))<\delta \varepsilon / 3$, where $\delta$ is the parameter of $\delta_{\lambda}$ BC integrability.

Choose now the following $D \in \Pi(F)$ : by means of Lyapunov theorem, divide $F$ into finitely many sets, say $I_{1}, \ldots, I_{k}$, each with $(\mu, \lambda)\left(I_{j}\right)=\left(x_{\widetilde{n}}^{\prime},(\lambda(F) / \mu(F)) x_{\widetilde{n}}^{\prime}\right)$, until $\mu\left(F \backslash \bigcup_{j=1}^{k} I_{j}\right) \leq x_{\tilde{n}}^{\prime}$ and then choose $I_{k+1}=F \backslash \bigcup_{j=1}^{k} I_{j}$; thus easily $\lambda\left(I_{k+1}\right)=(\lambda(F) / \mu(F)) \mu\left(I_{k+1}\right)$.

Then for $D=\left\{I_{1}, \ldots, I_{k}, I_{k+1}\right\}$ one has $\delta_{\lambda}(D)<\delta(\varepsilon / 3)$.

We have then, similar to the computation in Proposition 3:

$$
\begin{aligned}
& \sum_{I \in D}\left|f[\mu(I)]-\ell_{1} \mu(I)\right| \\
& \quad=\sum_{n=1}^{k}\left|f\left(x_{\tilde{n}}^{\prime}\right)-\ell_{1} x_{\tilde{n}}^{\prime}\right|+\left|f\left[\mu\left(I_{k+1}\right)\right]-\ell_{1} \mu\left(I_{k+1}\right)\right| \\
& \sum_{n=1}^{k}\left|f\left(x_{\tilde{n}}^{\prime}\right)-\ell_{1} x_{\tilde{n}}^{\prime}\right|+\left|f\left[\mu\left(I_{k+1}\right)\right]\right|+\left|\ell_{1}\right| x_{\tilde{k}}^{\prime} \\
& \quad=\sum_{n=1}^{k}\left|\frac{f\left(x_{\tilde{n}}^{\prime}\right)-\ell_{1} x_{\tilde{n}}^{\prime}}{x_{\tilde{n}}^{\prime}}\right| x_{\tilde{n}}^{\prime}+\frac{\varepsilon}{3}+\frac{\varepsilon}{3} .
\end{aligned}
$$

As for the first sum we have the following estimate:

$$
\begin{aligned}
\sum_{n=1}^{k}\left|\frac{f\left(x_{\tilde{n}}^{\prime}\right)-\ell_{1} x_{\tilde{n}}^{\prime}}{x_{\tilde{n}}^{\prime}}\right| x_{\tilde{n}}^{\prime} & <\frac{\varepsilon}{3 \mu(F)} \sum_{n=1}^{k} x_{\tilde{n}}^{\prime} \\
& =\frac{\varepsilon}{3} \cdot \frac{\mu\left(F \backslash I_{k+1}\right)}{\mu(F)}<\frac{\varepsilon}{3}
\end{aligned}
$$

In conclusion

$$
\sum_{I \in D}\left|f[\mu(I)]-\ell_{1} \mu(I)\right|<\varepsilon .
$$

Clearly we can repeat this construction with $x_{\tilde{n}}^{\prime \prime}$ and find another partition $D^{*} \in \Pi(F)$ with $\delta_{\lambda}\left(D^{*}\right)<\delta(\varepsilon / 3)$ as above; again

$$
\sum_{I \in D^{*}}\left|f[\mu(I)]-\ell_{2} \mu(I)\right|<\varepsilon
$$

It is then clear that, since $\ell_{1} \neq \ell_{2}$, the game $v$ is not $\delta_{\lambda}$-BC integrable.

Therefore, for instance, taking $f:[0,1] \rightarrow \mathbb{R}$ defined as

$$
f(x)= \begin{cases}x \sin \log x & \text { if } x \neq 0 \\ 0 & \text { if } x=0\end{cases}
$$

the game $v=f \circ \lambda \in A C_{\infty}$, since for $x \neq 0$ one has $f^{\prime}(x)=$ $\sin \log x+\cos \log x \in L^{\infty}$, but, as $f^{\prime}(0)$ does not exist, according to the previous result, $\nu \notin \mathrm{BC}$.
We will need in the sequel the following lemma.

Lemma 15. The space $A C_{\infty}$ is symmetric and the following equality holds for every $\vartheta \in \mathscr{G}$ :

$$
\left\|\vartheta_{*} \nu\right\|_{\infty}=\|\nu\|_{\infty} .
$$

Proof. Fix $\varepsilon>0$ and choose $\mu \in N A^{+}$such that $-\mu \preceq \nu \preceq \mu$ and $\mu(\Omega)<\|\nu\|_{\infty}+\varepsilon$.

Let $\lambda=\vartheta_{*} \mu \in N A^{+}$. If $A \subset B$, then $\vartheta(A) \subset \vartheta(B)$ and therefore, by monotonicity, $(\mu-\nu)[\vartheta(A)] \leq(\mu-\nu)[\vartheta(B)]$, or else $\vartheta_{*} \mu(A)-\vartheta_{*} \nu(A) \leq \vartheta_{*} \mu(B)-\vartheta_{*} \nu(B)$, which is the same as to say that $\vartheta_{*} \mu-\vartheta_{*} \nu$ is monotone, and hence $\vartheta_{*} \nu \preceq \vartheta_{*} \mu$.

In a completely analogous way, as $\vartheta_{*}(-\mu)=-\vartheta_{*}(\mu)$, one reaches $-\vartheta_{*}(\mu) \preceq \vartheta_{*}(\nu)$. In conclusion $-\lambda \preceq \vartheta_{*} \nu \preceq \lambda$.

Moreover, $\lambda(\Omega)=\vartheta_{*}[\mu(\Omega)]=\mu[\vartheta(\Omega)]=\mu(\Omega)$ whence

$$
\left\|\vartheta_{*} \nu\right\|_{\infty} \leq \lambda(\Omega)=\mu(\Omega)<\|\nu\|_{\infty}+\varepsilon .
$$

To prove the converse inequality, first of all, for $\lambda \in N A$, consider the game $\vartheta_{*}^{-1} \lambda$ defined in the following fashion: for every $B \in \Sigma$ set $A=\vartheta^{-1}(B) \in \Sigma$ and set

$$
\vartheta_{*}^{-1} \lambda(B)=\lambda(A)
$$

so that $\vartheta_{*}\left[\vartheta_{*}^{-1} \lambda\right]=\lambda$. It is a routine computation, based on the properties of $\vartheta$, to show that $\vartheta_{*}^{-1} \lambda$ is a countably additive measure as well.

Again fix $\varepsilon>0$ and choose $\lambda \in N A$ such that $-\lambda \preceq \vartheta_{*} \nu \preceq$ $\lambda$ and $\lambda(\Omega)<\left\|\vartheta_{*} \nu\right\|_{\infty}+\varepsilon$.

Take $\mu=\vartheta_{*}^{-1} \lambda$ defined above.

Now $\vartheta_{*} \nu \succeq-\lambda=-\vartheta_{*} \mu$ (or else $\vartheta_{*} \nu+\vartheta_{*} \mu$ monotone) implies in turn that $\nu+\mu$ is monotone too, and similarly $\nu \preceq \mu$.

Hence

$$
\|\nu\|_{\infty} \leq \mu(\Omega)=\lambda\left[\vartheta^{-1}(\Omega)\right]=\lambda(\Omega)<\left\|\vartheta_{*} \nu\right\|_{\infty}+\varepsilon
$$

which concludes the proof of relationship (33).

In $Q$ we have the following result.

Proposition 16. The BC integral $\partial_{\varnothing}^{+}$is a Milnor semivalue on Q.

Proof. Let $\nu$ be any game in $Q, \lambda \in D^{\nu}$; then $\lambda-v$ is a monotone game, and hence $(\lambda-\nu)(E) \geq 0$ for each $E \in \Sigma$, which in turn implies immediately that $\partial_{\varnothing}^{+}(\lambda-\nu) \geq 0$, namely, $\lambda-\partial_{\varnothing}^{+}(\nu) \geq 0$ setwise in $\Sigma$. As $\lambda-\partial_{\varnothing}^{+}(\nu)$ is a measure, this equivalently says that $\lambda-\partial_{\varnothing}^{+}(\nu)$ is monotone, that is, $\lambda \preceq \partial_{\varnothing}^{+}(\nu)$. In complete analogy if $\lambda \in D_{\nu}$, then $-\lambda \preceq \partial_{\varnothing}^{+}(\nu)$.

Hence for $\nu \in Q$ we have necessarily $\nu_{*} \leq \partial_{\varnothing}^{+}(\nu) \leq \nu^{*}$ which proves that $\partial_{\varnothing}^{+}$is a MO.

Finally, we deduce from (11) the symmetry of the operator $\partial_{\varnothing}^{+}$, and the proof is thus complete.

According to Theorem 1.8 in [9], $\partial_{\varnothing}^{+}$can be extended to the whole space $A C_{\infty}$ in such a way that the extension, which we will label as $\widetilde{\partial_{\varnothing}^{+}}$, remains a linear MO. Recall moreover that 
MO on subspaces of $A C_{\infty}$ are continuous with respect to the norm $\|\cdot\|_{\infty}[9$, Lemma 1.6].

Let $Q_{\infty}$ denote the $\|\cdot\|_{\infty}$-closure of $Q$. Then on $Q_{\infty}$ we have the following.

Theorem 17. $\widetilde{\partial_{\varnothing}^{+}}$is a Milnor semivalue on $Q_{\infty}$.

Proof. If $v \in Q_{\infty}$, there exists a sequence in $Q$, say $\left(v_{k}\right)_{k}$ that $\|\cdot\|_{\infty}$ converges to $\nu$.

Because of (33), for each $\vartheta \in \mathscr{G}$, we have that $\vartheta_{*} \nu_{k} \stackrel{\|\cdot\|_{\infty}}{\longrightarrow}$ $\vartheta_{*} \nu$. But then $\partial_{\varnothing}^{+}\left(\vartheta_{*} \nu_{k}\right) \stackrel{\|\cdot\|_{\infty}}{\longrightarrow} \widetilde{\partial_{\varnothing}^{+}}\left(\vartheta_{*} \nu\right)$ too.

Similarly $\partial_{\varnothing}^{+}\left(\nu_{k}\right) \stackrel{\|\cdot\|_{\infty}}{\longrightarrow} \widetilde{\partial_{\varnothing}^{+}}(\nu)$, and then, again by (33), $\vartheta_{*}\left[\partial_{\varnothing}^{+}\left(\nu_{k}\right)\right] \stackrel{\|\cdot\|_{\infty}}{\longrightarrow} \vartheta_{*}\left[\widetilde{\partial_{\varnothing}^{+}}(\nu)\right]$.

In conclusion $\vartheta_{*}\left[\widetilde{\partial_{\varnothing}^{+}}(\nu)\right]=\widetilde{\partial_{\varnothing}^{+}}\left(\vartheta_{*} \nu\right)$.

Since powers of probabilities belong to $Q$, there immediately follows the following.

Corollary 18. $\widetilde{\partial_{\emptyset}^{+}}$is a $\|\cdot\|_{\infty}$-continuous semivalue on $p N A_{\infty}$.

We point out that, as $Q, Q_{\infty}$, and $p N A_{\infty}$ are symmetric subspaces of $A C_{\infty}$, there follows from [9, Theorem 3.1] that $\partial_{\varnothing}^{+}$and $\widetilde{\partial_{\varnothing}^{+}}$are diagonal.

Moreover from [9, Theorem 2.1], there exists a Borel measure $\xi$ on $[0,1]$ such that the following representation of $\widetilde{\partial_{\varnothing}^{+}}$on $p N A_{\infty}$ holds:

$$
\widetilde{\partial_{\varnothing}^{+}}(\nu, S)=\int_{0}^{1} \partial \nu^{*}\left(t \mathbf{1}_{\Omega}, \mathbf{1}_{S}\right) d \xi, \quad S \in \Sigma,
$$

where $\partial \nu^{*}$ is the ideal extension of the game $\nu$ defined in $[1$, Theorem G].

Define now the space FEAS $1=Q \cap F E A S$. Note that $F E A S_{1}=Q \cap F E A S_{0}$. Also define

$$
\text { FEAS }_{\infty}=\left\{\nu \in Q_{\infty}: \widetilde{\partial_{\varnothing}^{+}}(\nu, \Omega)=\nu(\Omega)\right\}
$$

Proposition 19. FEAS $S_{\infty}$ is the $\|\cdot\|_{\infty}$-closure of FEAS .

Proof. Denote by $[Q \cap F E A S]_{\infty}$ the $\|\cdot\|_{\infty}$-closure of FEAS $S_{1}$. Indeed it is immediate to prove that $[Q \cap F E A S]_{\infty} \subset F E A S_{\infty}$ : take $v \in[Q \cap F E A S]_{\infty}$ and a sequence $\left(\nu_{k}\right)_{k} \in F E A S_{1}$ converging in the $\|\cdot\|_{\infty}$ norm to $\nu$. Then $\nu \in Q_{\infty}$. Moreover, the convergence of $\left(\nu_{\mathrm{k}}\right)_{k}$ to $\nu$ implies that $\nu_{k}(\Omega)$ converges to $\nu(\Omega)$. Furthermore, $\partial_{\varnothing}^{+}\left(\nu_{k}, \cdot\right)$ converges in variation (and hence setwise) to $\widetilde{\partial_{\varnothing}^{+}}(\nu, \cdot)$. Therefore,

$$
\nu(\Omega)=\lim _{k} \nu_{k}(\Omega)=\lim _{k} \partial_{\varnothing}^{+}\left(\nu_{k}, \Omega\right)=\widetilde{\partial_{\varnothing}^{+}}(\nu, \Omega),
$$

so $v \in F E A S_{\infty}$.

For the converse inclusion, take $v \in F E A S_{\infty}$. Hence $v$ is the $\|\cdot\|_{\infty}$-norm limit of a sequence $\left(\nu_{k}\right)_{k}$ of elements in $Q$. Construct now the sequence

$$
\tau_{k}=v_{k}+\left[\partial_{\varnothing}^{+}\left(v_{k}, \Omega\right)-v_{k}(\Omega)\right] \mu^{2},
$$

for some $\mu \in N A^{1}$. We claim that $\tau_{k} \in F E A S_{1}$ and $\tau_{k}\|\cdot\|_{\infty}$ converges to $\nu$.

Indeed, by the feasibility of $v$ and by the fact that $\nu_{k}(\Omega) \rightarrow \nu(\Omega)$ and $\partial_{\varnothing}^{+}\left(\nu_{k}, \Omega\right) \rightarrow \widetilde{\partial_{\varnothing}^{+}}(\nu, \Omega)$, it follows that $\tau_{k}\|\cdot\|_{\infty}$ converges to $\nu$. Moreover, as $\partial_{\varnothing}^{+}\left(\tau_{k}, \cdot\right)=\partial_{\varnothing}^{+}\left(\nu_{k}, \cdot\right)$, it immediately follows that $\tau_{k} \in$ FEAS.

Obviously $\widetilde{\partial_{\varnothing}^{+}}$is a value of $F E A S_{\infty}$, but, unfortunately, on the subspace $p N A_{\infty} \cap F E A S_{\infty}$ we lose uniqueness, in that $\widetilde{\partial_{\emptyset}^{+}}$ does not agree with the Aumann-Shapley (AS) value.

Example 20. Let $f(x, y)=\phi_{1}(x)+\phi_{2}(y)$, where $\phi_{1}(x)=$ $x^{2} / 2+x / 2$ and $\phi_{1}(y)=-\left(y^{2} / 2\right)+y$. Let $\mu_{1}$ and $\mu_{2}$ be two linearly independent measures in $N A^{1}$, and $\nu=f \circ \mu$, where $\mu=\left(\mu_{1}, \mu_{2}\right)$.

We claim that $v \in F E A S_{\infty}$, but $\widetilde{\partial_{\varnothing}^{+}}(\nu) \neq \Phi(\nu)$, where $\Phi$ denotes the AS value.

The function $f \in C^{1}\left(\mathbb{R}^{2}\right)$ and we know that $\Phi(\nu)=$ $\left[\int_{0}^{1} f_{x}^{\prime}(t, t) d t\right] \mu_{1}+\left[\int_{0}^{1} f_{y}^{\prime}(t, t) d t\right] \mu_{2}$ and $\partial_{\varnothing}^{+}(\nu)=f_{x}^{\prime}(0) \mu_{1}+$ $f_{y}^{\prime}(0) \mu_{2}$. Hence $\widetilde{\partial_{\varnothing}^{+}}(\nu)=\left(\mu_{1} / 2\right)+\mu_{2}$, while $\Phi(\nu)=\mu_{1}+\mu_{2} / 2$. It is also immediate to check that $\nu \in F E A S_{\infty}$.

Indeed one can provide infinitely many examples of subspaces of $p N A_{\infty}$ where a value different from the AS one is defined. By means of [9, Theorem 2.1], each probability measure $\xi$ on $[0,1]$ generates a Milnor semivalue $\pi_{\xi}$ on $p N A_{\infty}$ which clearly becomes a value on the subspace FEAS $_{\xi}=\left\{\nu \in p N A_{\infty}: \pi_{\xi}(\nu)(\Omega)=\nu(\Omega)\right\}$. In our case for the Dirac measure $\delta_{0}$ based at 0 , we have precisely $F E A S_{\delta_{0}}=p N A_{\infty} \cap F E A S_{\infty}$. However, we point out that our main interest in this paper is not uniqueness of the value but the fact that the Burkill-Cesari integral and hence the Epstein-Marinacci derivative constitute a value.

It may anyway be of interest to characterize games in $p N A_{\infty} \cap F E A S_{\infty}$. Remember that $p N A_{\infty}$ is the $\|\cdot\|_{\infty}$-closure of the linear span $B$ of powers of nonatomic probability measures. With the same technique used in Proposition 19 one proves that $p N A_{\infty} \cap F E A S_{\infty}$ is in fact the $\|\cdot\|_{\infty}$-closure of $B \cap F E A S_{0}$. It is now easy to characterize these games: in fact if $\nu=\sum_{k=1}^{n} \alpha_{k} \mu_{k}^{k} \in B \cap F E A S_{0}$, with $\mu_{k} \in N A^{+}$, then $\partial_{\varnothing}^{+}(\nu, \cdot)=$ $\alpha_{1} \mu_{1}$. So $\nu \in F E A S_{0}$ if and only if $\sum_{k=2}^{n} \alpha_{k}\left[\mu_{k}(\Omega)^{k}\right]=0$.

\section{Appendix}

We will first prove that the measure game in Example 9 is in AC.

Let $I_{1}=\left[-1,-\alpha\left[, I_{2}=[-\alpha, \alpha], I_{3}=\right] \alpha, 1\right]$ and let $m=|\mu|$ (the total variation of $\mu$ ).

Fix a Hahn decomposition $(P, N)$ of $\mu$.

We will prove that $v \ll m$.

Fix $\varepsilon>0$ and choose $\delta=\varepsilon \wedge \alpha$. Let $C$ be a chain and $\Lambda$ a subchain with $\|m\|_{\Lambda}<\delta$. We claim that $\|\nu\|_{\Lambda}<\varepsilon$.

In fact, let $\left(S_{i}, S_{i+1}\right)$ be a link in $\Lambda$. Then, as $m\left(S_{i+1}\right)-$ $m\left(S_{i}\right)<\alpha$, necessarily if $\mu\left(S_{i}\right), \mu\left(S_{i+1}\right)$ do not belong to the same $I_{j}$, then they are at most in two contiguous $I_{j}$ 's.

Indeed, suppose, for instance, that $\mu\left(S_{i}\right) \in I_{1}, \mu\left(S_{i+1}\right) \in I_{3}$, namely, $\mu\left(S_{i}\right)<-\alpha, \mu\left(S_{i+1}\right)>\alpha$. Hence $\mu\left(S_{i} \cap P\right)+\mu(\operatorname{Si\cap N})=$ 
$m\left(S_{i} \cap P\right)-m\left(S_{i} \cap N\right)<-\alpha$ and analogously $m\left(S_{i+1} \cap P\right)-$ $m\left(S_{i+1} \cap N\right)>\alpha$. Thus

$$
\begin{aligned}
& {\left[m\left(S_{i+1} \cap P\right)-m\left(S_{i+1} \cap N\right)\right]} \\
& \quad-\left[m\left(S_{i} \cap P\right)-m\left(S_{i} \cap N\right)\right]>2 \alpha
\end{aligned}
$$

that is;

$$
\begin{aligned}
& {\left[m\left(S_{i+1} \cap P\right)-m\left(S_{i} \cap P\right)\right]} \\
& \quad-\left[m\left(S_{i+1} \cap N\right)-m\left(S_{i} \cap N\right)\right]>2 \alpha
\end{aligned}
$$

namely,

$$
\left[m\left(S_{i+1} \backslash S_{i}\right) \cap P\right]-\left[m\left(S_{i+1} \backslash S_{i}\right) \cap N\right]>2 \alpha
$$

whence, a fortiori, $m\left(S_{i+1} \backslash S_{i}\right)>2 \alpha$ which contradicts the initial assumption $\|m\|_{\Lambda}<\alpha$.

Then an easy computation shows that in both cases (either $\mu\left(S_{i}\right), \mu\left(S_{i+1}\right)$ belong to the same $I_{j}$ or they lie in two contiguous $I_{j}$ 's)

$$
\left|\nu\left(S_{i+1}\right)-v\left(S_{i}\right)\right| \leq\left|\alpha m\left(S_{i+1}\right)-\alpha m\left(S_{i}\right)\right|
$$

and this proves our claim, since $\alpha<1$.

In a completely analogous way one shows that the game $v$ in Example 10 is in $A C$.

To prove that $\Psi: p N A E \rightarrow N A$ in the same example is a value, the only property that needs to be checked is positivity (the others are obvious).

Assume that $\gamma=\sum_{i=1}^{m} c_{i} \vartheta_{i}^{*} w+p \in p N A E, p \in p N A$ is monotone.

We will also use the alternative form $\gamma=\sum_{i=1}^{m} c_{i} \vartheta_{i}^{*} \nu+u$, where $u=\sum_{i=1}^{m} c_{i} \vartheta_{i}^{*} \lambda+p$.

Now by (11)

$$
\begin{aligned}
\Psi(\gamma) & =\sum_{i=1}^{m} c_{i} \partial_{\varnothing}^{+}\left(\vartheta_{i}^{*} w, \cdot\right)+\phi_{\mathrm{AS}}(p) \\
& =\sum_{i=1}^{m} c_{i} \vartheta_{i}^{*} \lambda+\phi_{\mathrm{AS}}(p)=\phi_{\mathrm{AS}}(u)
\end{aligned}
$$

since $\partial_{\varnothing}^{+}(\nu, \cdot)=0$, while $\partial_{\varnothing}^{+}(\lambda, \cdot)=\lambda$. Hence we need to prove that $\phi_{\mathrm{AS}}(u) \geq 0$.

Note first that since $v \in p N A^{\prime}$, the ideal set function $\nu^{*}$ exists, and it actually coincides with $f \circ \mu^{*}[1$, Proposition 22.16 p. 152], and that $\mu^{*}(\varphi)=\int_{\Omega} \varphi d \mu$. Hence the ideal function $\gamma^{*}$ exists too.

Recall now the definition of $\partial v_{+}^{*}(t, S), \partial v_{-}^{*}(t, S), t \in$ $[0,1], S \in \sum$ from [16].

In particular, since

$$
\begin{gathered}
\partial \nu_{+}^{*}(t, S)=\lim _{\tau \downarrow 0} \frac{\nu^{*}\left(t \mathbf{1}_{\Omega}+\tau \mathbf{1}_{S}\right)-\nu^{*}\left(t \mathbf{1}_{\Omega}\right)}{\tau}, \\
\nu^{*}\left(t \mathbf{1}_{\Omega}+\tau \mathbf{1}_{S}\right)-\nu^{*}\left(t \mathbf{1}_{\Omega}\right) \\
=f\left[t \int_{\Omega} \mathbf{1}_{\Omega} d \mu+\tau \int_{\Omega} \mathbf{1}_{S} d \mu\right]-f\left[t \int_{\Omega} \mathbf{1}_{\Omega} d \mu\right] \\
=f[t \mu(\Omega)+\tau \mu(S)]-f[t \mu(\Omega)]=f[\tau \mu(S)]
\end{gathered}
$$

(for $\mu(\Omega)=0$ ), we find

$$
\partial \nu_{+}^{*}(t, S)=\lim _{\tau \downarrow 0} \frac{f[\tau \mu(S)]}{\tau}=f^{\prime}(0) \mu(S)=0
$$

and similarly for the left-hand side limit. In other words $\partial \nu^{*}(t, S)=0$ for each $t, S$.

We note now that, since $v$ is a measure game, for every $\vartheta \in \mathscr{G}, \partial\left(\vartheta^{*} \nu\right)_{+}^{*}=\vartheta^{*} \partial \nu_{+}^{*}=0$ [1, Proposition 22.16, p. 152], and the proof of Theorem 3 in [16]).

Now, since $\gamma$ is monotone, both $\partial \gamma_{+}^{*}(t, S), \partial \gamma_{-}^{*}(t, S)$ are nonnegative for each $t, S$. Hence

$$
\begin{aligned}
0 & \leq \partial \gamma_{+}^{*}(t, S)=\sum_{i=1}^{m} c_{i} \partial\left(\vartheta_{i}^{*} \nu\right)_{+}^{*}(t, S)+\partial u^{*}(t, S) \\
& =\partial u^{*}(t, S),
\end{aligned}
$$

where in the last summand we do not need to distinguish between $\partial u_{+}^{*}$ and $\partial u_{-}^{*}$ as $u \in p N A$ and hence $\partial u^{*}$ exists.

But then

$$
0 \leq \int_{0}^{1} \partial u^{*}(t, S) d t=\phi_{\mathrm{AS}}(u)(S), \quad S \in \Sigma,
$$

which is precisely what we wanted to prove.

\section{Conflict of Interests}

The authors declare that there is no conflict of interests regarding the publication of this paper.

\section{References}

[1] R. J. Aumann and L. S. Shapley, Values of Non-Atomic Games, Princeton University Press, Princeton, NJ, USA, 1974.

[2] L. G. Epstein and M. Marinacci, "The core of large differentiable TU games," Journal of Economic Theory, vol. 100, no. 2, pp. 235273, 2001.

[3] J.-F. Mertens, "Values and derivatives," Mathematics of Operations Research, vol. 5, no. 4, pp. 523-552, 1980.

[4] L. Montrucchio and P. Semeraro, "Refinement derivatives and values of games," Mathematics of Operations Research, vol. 33, no. 1, pp. 97-118, 2008.

[5] S. Hart and A. Mas-Colell, "Potential, value, and consistency," Econometrica, vol. 57, no. 3, pp. 589-614, 1989.

[6] F. Centrone and A. Martellotti, "A mesh-based notion of differential for TU games," Journal of Mathematical Analysis and Applications, vol. 389, no. 2, pp. 1323-1343, 2012.

[7] P. Dubey, A. Neyman, and R. J. Weber, "Value theory without efficiency," Mathematics of Operations Research, vol. 6, no. 1, pp. 122-128, 1981.

[8] S. Hart and D. Monderer, "Potentials and weighted values of nonatomic games," Mathematics of Operations Research, vol. 22, no. 3, pp. 619-630, 1997.

[9] D. Monderer, "A Milnor condition for nonatomic Lipschitz games and its applications," Mathematics of Operations Research, vol. 15, no. 4, pp. 714-723, 1990.

[10] D. Candeloro and A. Martellotti, "Geometric properties of the range of two-dimensional quasi-measures with respect to the Radon-Nikodym property," Advances in Mathematics, vol. 93, no. 1, pp. 9-24, 1992. 
[11] L. Cesari, "Quasi-additive set functions and the concept of integral over a variety," Transactions of the American Mathematical Society, vol. 102, pp. 94-113, 1962.

[12] A. Ghizzetti and A. Ossicini, Polinomi Ortogonali e Problema dei Momenti, Istituto per le Applicazioni del Calcolo Mauro Picone, Rome, Italy, 1981.

[13] E. Kohlberg, "On non-atomic games: conditions for $f \circ \mu \epsilon$ pNA," International Journal of Game Theory, vol. 2, pp. 87-98, 1973.

[14] M. Marinacci and L. Montrucchio, "Introduction to the mathematics of ambiguity," in Uncertainty in Economic Theory, I. Gilboa, Ed., pp. 46-107, Routledge, New York, NY, USA, 2004.

[15] A. Martellotti, "Core equivalence theorem: countably many types of agents and commodities in $L^{1}(\mu)$," Decisions in Economics and Finance, vol. 30, no. 1, pp. 51-70, 2007.

[16] Y. Tauman, "Value on a class of nondifferentiable market games," International Journal of Game Theory, vol. 10, no. 3-4, pp. 155-162, 1981. 


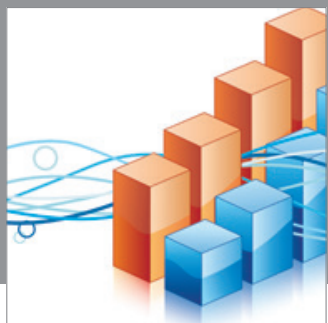

Advances in

Operations Research

mansans

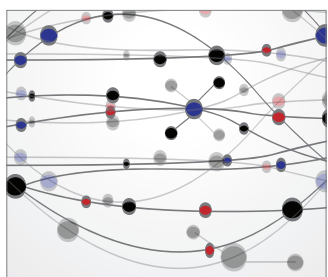

The Scientific World Journal
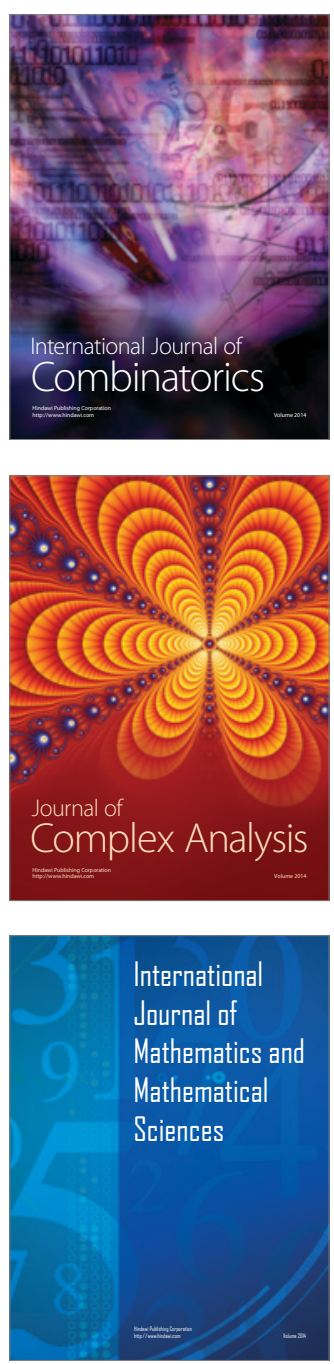
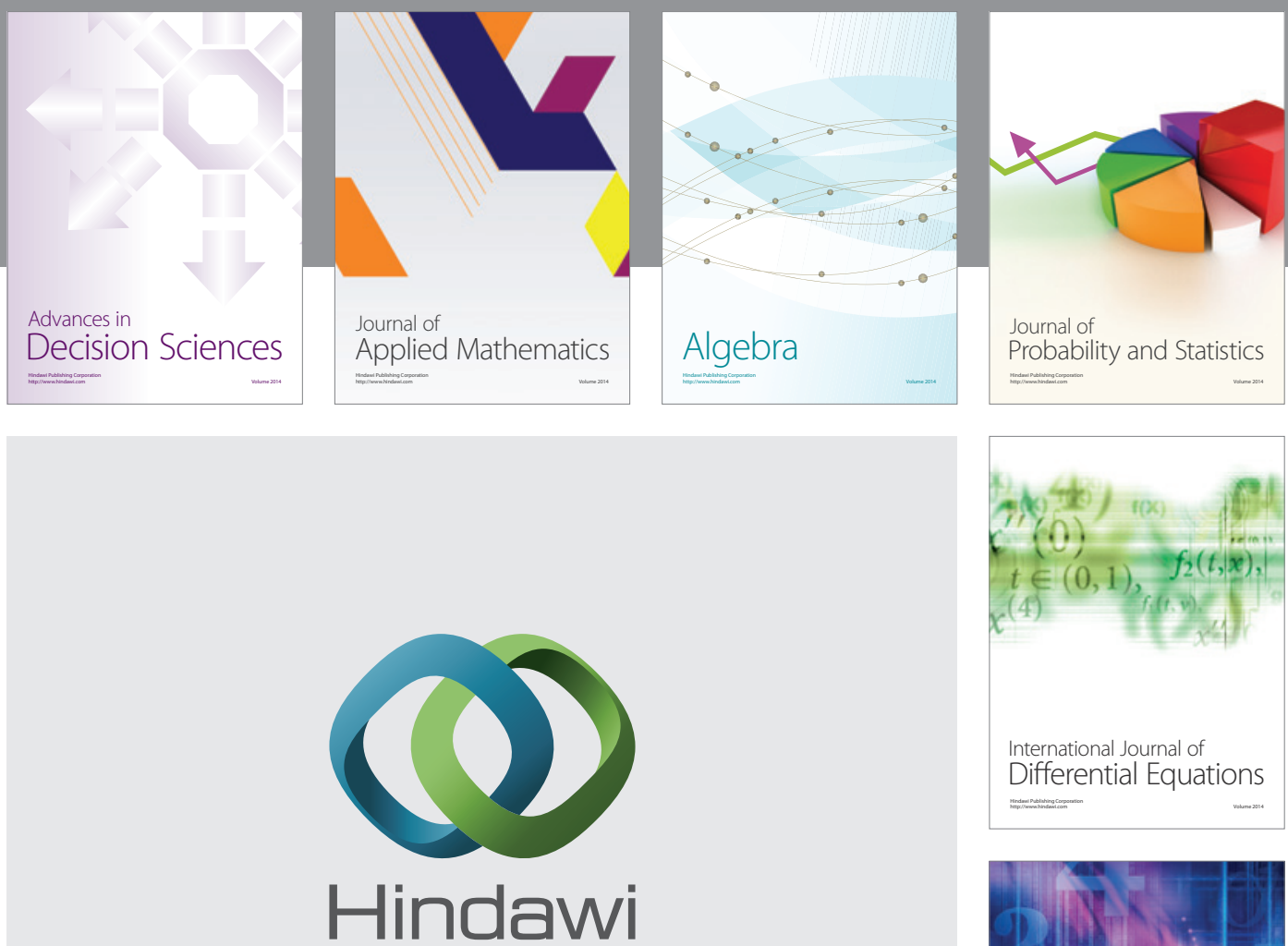

Submit your manuscripts at http://www.hindawi.com
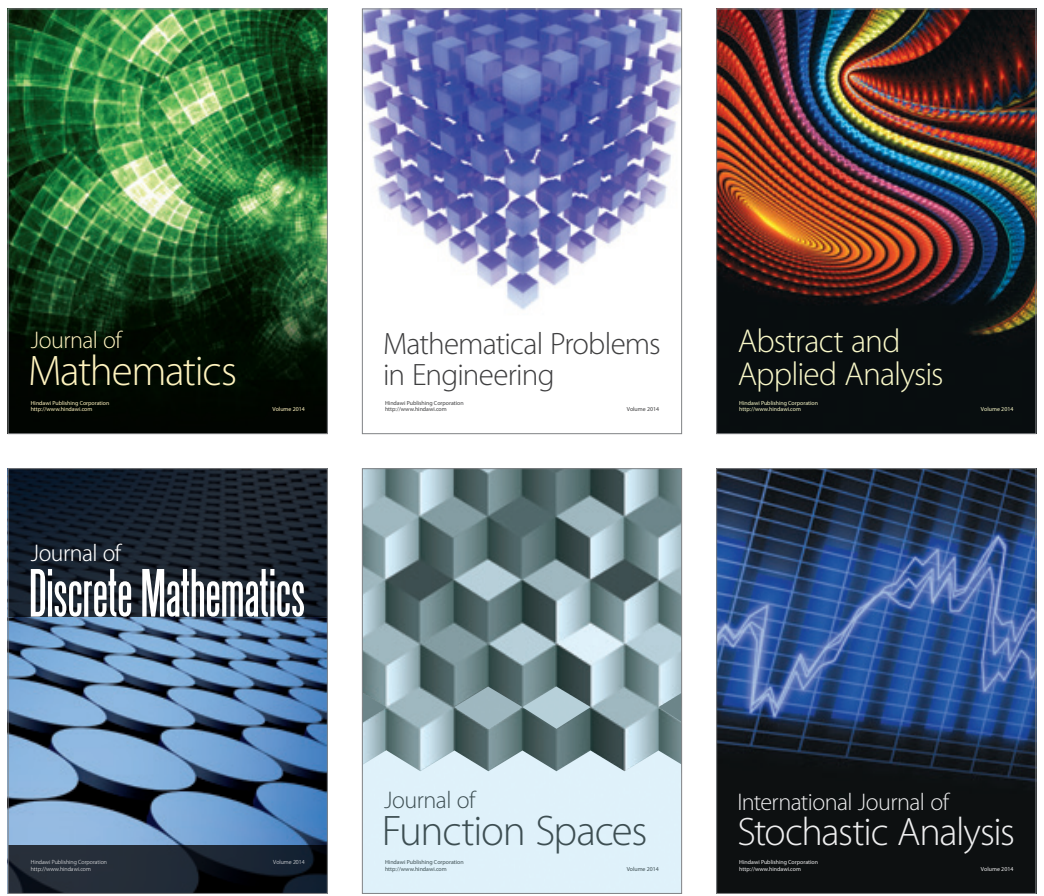

Journal of

Function Spaces

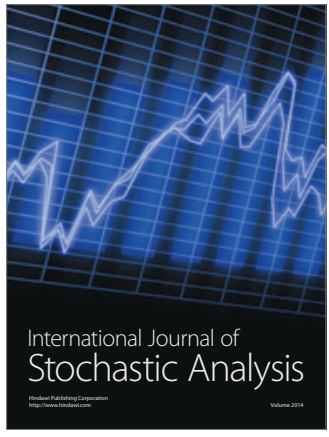

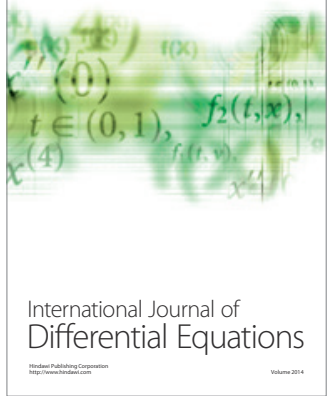
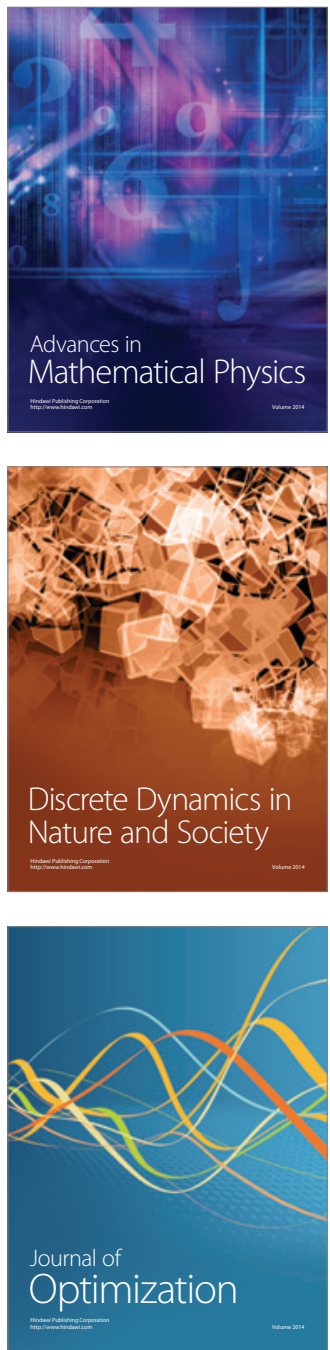